\title{
The Genetics Corner: Pathogenic variants in DOCK6 mimic congenital viral infection in an SGA infant with VSD and intracranial calcifications
}

Subhadra Ramanathan, Robin D. Clark, MD

\section{Case Summary:}

This baby girl was born to an 18-year old primigravida at 35 weeks three days' gestation by planned induction of labor for oligohydramnios and IUGR. Fetal ultrasound examination revealed a single umbilical artery and a VSD. Mother had irritable bowel syndrome with abdominal pain and vomiting during pregnancy treated with amitriptyline, omeprazole (Prilosec), ondansetron $\mathrm{HCl}$ (Zofran) until two months gestation. She denied teratogenic exposures. Apgar scores were 7 and 9 . Birth weight $1925 \mathrm{~g}\left(9^{\text {th }}\right.$ $\%$ ile), length $40.1 \mathrm{~cm}\left(<3^{\text {rd }} \%\right.$ ile $)$, head circumference $30 \mathrm{~cm}\left(11^{\text {th }}\right.$ $\%$ ile). The placenta was small (266 grams, $<3$ rd \%ile) with mild decidual arteriopathy but no infarctions or significant inflammatory changes.

The initial physical exam revealed mild micrognathia, a loud 2/6 holosystolic murmur, bilateral sandal gaps between the first and second toes, and a deep groove on the plantar surface of each foot. She had a high-pitched shrill cry and unusual posturing of the extremities: wrists flexed, and hands were held above the shoulders, ankles crossed, with generalized hypertonia and clonus at the ankles.

There was a large perimembranous type VSD with a left ventricle to right atrial shunt and a persistent superior vena cava on echocardiogram. The brain MRI revealed "mildly enlarged lateral ventricular bodies and atria with periventricular subependymal foci of T1 shortening suggesting calcification and scattered subarachnoid hemorrhage versus areas cortical calcification. Findings are consistent with intrauterine TORCH infection." A dilated eye exam by the ophthalmology consultant was normal. Chromosome microarray, Zika and TORCH titers, serum PCR for Zika, and urine PCR for CMV were negative.

The family history was significant for VSD and atrial fibrillation in the paternal grandmother, paternal great grandfather, and paternal great uncle (paternal grandmother's brother). The father had ADHD and bipolar disorder.

Because of the baby's abnormal neuro exam and CNS calcifications, the initial plan to order a cardiac gene panel was changed to a whole-exome sequencing test (Baylor). This test revealed two likely pathogenic variants in DOCK6, the gene responsible for the autosomal recessive Adams-Oliver syndrome 2 (OMIM 614219). The infant had two variants that caused premature truncation of the gene product: a novel variant c.3737_3738del (p.S1246Cfs*2) and c.1987C>T (p.Q663*).

At a return outpatient visit a four months of age, the VSD appeared to be closing spontaneously. The baby was less irritable, and her resting tone was normal without unusual posturing of the extremities. She had an area of scalp alopecia that had not been appreciated during the newborn exam. There were no digital anomalies except mild syndactyly of toes 2 and 3 and the previously noted sandal gap.

The mother provided an additional family history that the father also had an area of scalp alopecia at the vertex.

\section{Discussion:}

This child, who was small for gestational age, had features that suggested a congenital viral infection: a septal heart defect, an abnormal neurological examination, and intracranial calcifications. When viral titers and PCR tests were negative, whole-exome sequencing revealed two likely pathogenic variants in DOCK6. This gene is responsible for the autosomal recessive condition, AdamsOliver syndrome 2 (AOS2, OMIM 614219).

Adams and Oliver described their eponymous syndrome in 1945 (AOS, OMIM 100300) as a phenotype comprised primarily of aplasia cutis congenita (ACC) and transverse terminal defects of the limbs. AOS is now considered a heterogeneous disorder with at least six responsible genes: AOS1, 3, 5, and 6 are autosomal dominant, and AOS2 and AOS4 are autosomal recessive traits.

\section{"AOS was initially thought to be a disorder of vascular neogenesis, but evidence now points to genes that impair the actin cytoskeleton"s formation."}

AOS was initially thought to be a disorder of vascular neogenesis, but evidence now points to genes that impair the actin cytoskeleton's formation. DOCK6, the dedicator of cytokinesis $\underline{6}$ gene, encodes an atypical guanidine exchange factor (GEF) known to activate two members of the Rho GTPase family of signaling proteins: Cdc42 and Rac1. Both Cdc42 and Rac1 induce actin polymerization in the cytoskeleton. Shaheen et al. (2011) have shown that the DOCK6 mRNA expression profile is consistent with the AOS2 phenotype with expression in the distal developing limb bud in the mouse. Inactivating mutations in DOCK6 are likely to impair actincytoskeleton organization, but the mechanism of action responsible for alopecia, cardiac defects, and intracranial calcifications has not been elucidated.

In AOS, the ACC is usually in the midline, parietal or occipital region of the scalp. Structures underlying these skin defects (skull bones, meninges, sinus) may also be involved. The transverse terminal limb defects are typically limb truncation defects affecting the distal phalanges or entire digits (true ectrodactyly). Syndactyly, most commonly of second and third toes, can also be seen. Interestingly, this child has an uncommon and milder presentation of the limb defects associated with AOS, but not all patients with AOS have transverse limb defects (Dudoignon et al. 2020). To complicate matters, our patient's scalp defect was initially missed, so none of the classic features of AOS were evident that would have suggested the diagnosis.

The clinical features of AOS are highly variable and include cardio-

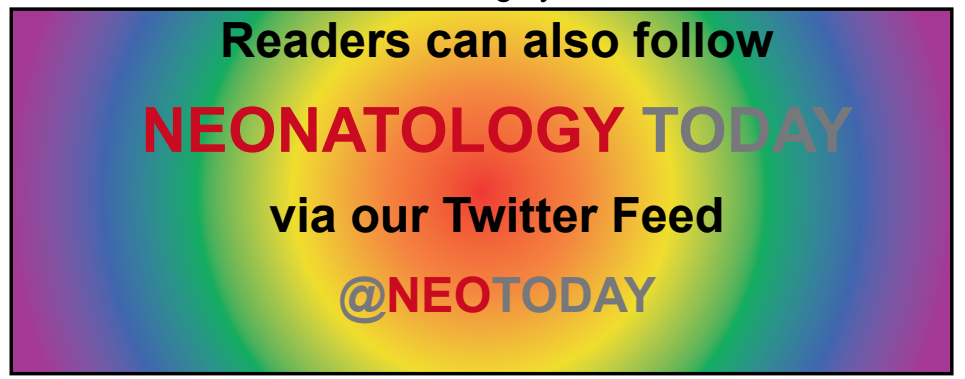



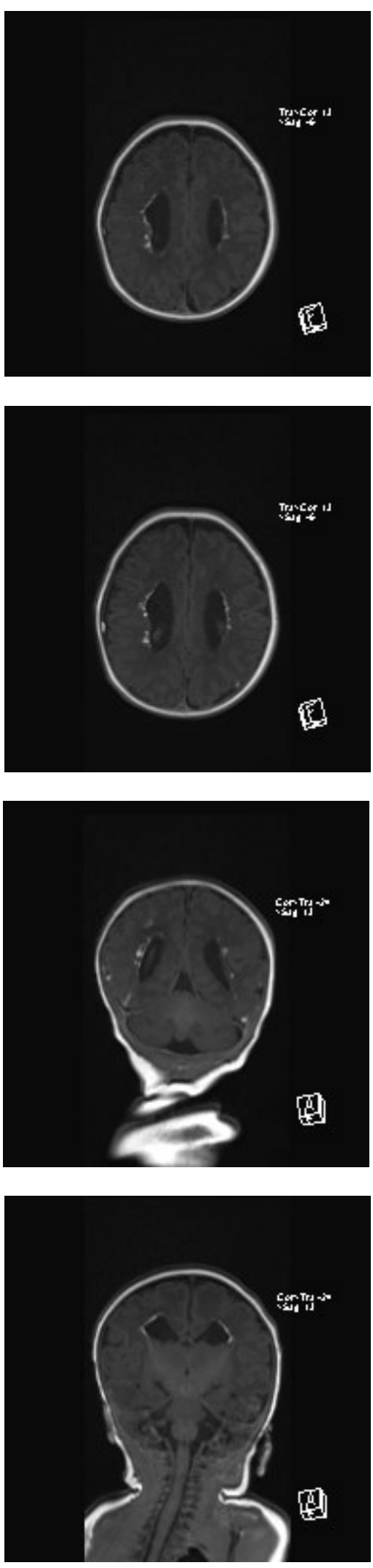

vascular malformations, brain abnormalities, and vascular defects such as cutis marmorata and dilated scalp veins. Our patient is one of the about $20 \%$ of patients with AOS who have congenital heart defects, including septal defects (Snape et al. 2008). Dudoignon and colleagues (2020) reviewed the clinical features of 29 patients with AOS, including three with variants in DOCK6, and their patient nine who had intrauterine growth retardation, intracranial calcifications, and cardiac anomalies (valvulopathy) was similar to our patient.

Sukalo et al. (2015) described ten patients with biallelic pathogenic variants in DOCK 6 and abnormal structural brain anomalies. The most common CNS anomalies were ventriculomegaly, periventricular leukomalacia, calcifications, and hypoplasia or atrophy of the corpus callosum. They described developmental delay and seizures. Ocular findings associated with AOS2 include optic nerve hypoplasia, microphthalmia, retinal detachment. Sukalo and colleagues conclude that DOCK6 mutations are "strongly associated with structural brain abnormalities, ocular anomalies, and intellectual disability, thus suggesting that DOCK6-linked disease represents a variant of AOS with a particularly poor prognosis."

Both parents should be tested specifically for these two DOCK6 variants in order to establish the phase of the variants as cis or trans: when the phase is $c i, s$ both variants are inherited from the same parent on the same chromosome, whereas in trans, the variants are inherited on separate chromosomes, one from each parent. Parental testing will establish the biallelic nature of these variants in this patient and allow informed counseling regarding recurrence risks for future offspring born to these parents.

Practical Applications:

1. Select gene testing that is appropriate to the patient Whole exome sequencing was the better option and more appropriate than a cardiac gene panel for this infant with a congenital cardiac anomaly who also had poor overall growth and evidence of major CNS anomalies.

2. Consider a genetic etiology for the small for gestational age infant with intracranial calcifications when congenital infections such as TORCH and Zika have been ruled out.

3. Examine each infant carefully for minor anomalies, in this case, alopecia of the scalp, which was initially missed.

a. Minor anomalies can define the phenotype and guide testing choices.

b. Had we identified the infant's scalp defect prior to ordering gene testing, we might have chosen a more focused and less costly gene panel that was specific for Adams-Oliver syndrome.

4. Examine the parents and ask for information about even seemingly minor anomalies.

\section{References:}

1. Sukalo M, Tilsen F, Kayserili H, et al. DOCK6 mutations are responsible for a distinct autosomal-recessive variant of $\mathrm{Ad}$ ams-Oliver syndrome associated with brain and eye anomalies. Hum Mutat. 2015 Jun;36(6):593-8. Erratum in: Hum Mutat. 2015 Nov;36(11):1112. PMID: 25824905.

2. Shaheen $R$, Faqeih $E$, Sunker $A$, et al. Recessive mutations in DOCK6, encoding the guanidine nucleotide exchange factor DOCK6, lead to abnormal actin cytoskeleton organization and Adams-Oliver syndrome. Am J Hum Genet. 2011 Aug 12;89(2):328-33. PMID: 21820096

3. Snape KM, Ruddy $D$, Zenker $M$, et al. The spectra of clinical phenotypes in aplasia cutis congenita and terminal transverse limb defects. Am J Med Genet A. 2009 Aug;149A(8):1860-81. PMID: 19610107

4. Dudoignon $B$, Huber $C$, Michot $C$, et al. Expanding the phenotype in Adams-Oliver syndrome correlating with the genotype. Am J Med Genet A. 2020 Jan;182(1):29-37. PMID: 31654484

5. Adams-Oliver syndrome 2. On line Mendelian Inheritance in Man \#614219 https://omim.org/entry/614219?search=6142 $\underline{19 \& h i g h l i g h t=614219}$

Disclosures: The authors have no relevant disclosures.

\section{NT}

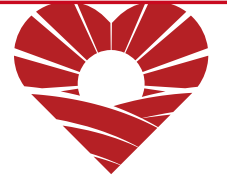

CONGENITAL CARDIOLOGY TODAY
The only worldwide monthly publication exclusively serving Pediatric and Adult Cardiologists that focus on Congenital/ Structural Heart Disease (CHD), and Cardiothoracic Surgeons.

\section{Subscribe Electronically} Free on the Home Page www.CongenitalCardiologyToday.com 


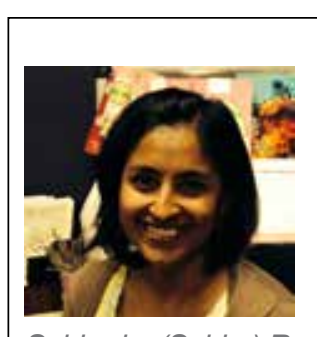

Subhadra (Subha) Ramanathan, M.Sc., M.S. Licensed and Certified Genetic Counselor Assistant Professor, Pediatrics Loma Linda University Health 2195 Club Center Drive, Ste A San Bernardino, CA 92408

SRamanathan@llu.edu
Corresponding Author

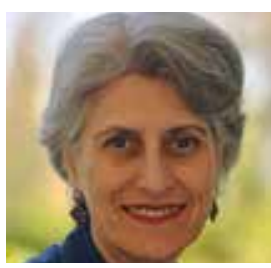

Robin Clark, MD

Professor, Pediatrics

Loma Linda University School of Medicine

Division of Genetics

Department of Pediatrics

Email rclark@llu.edu

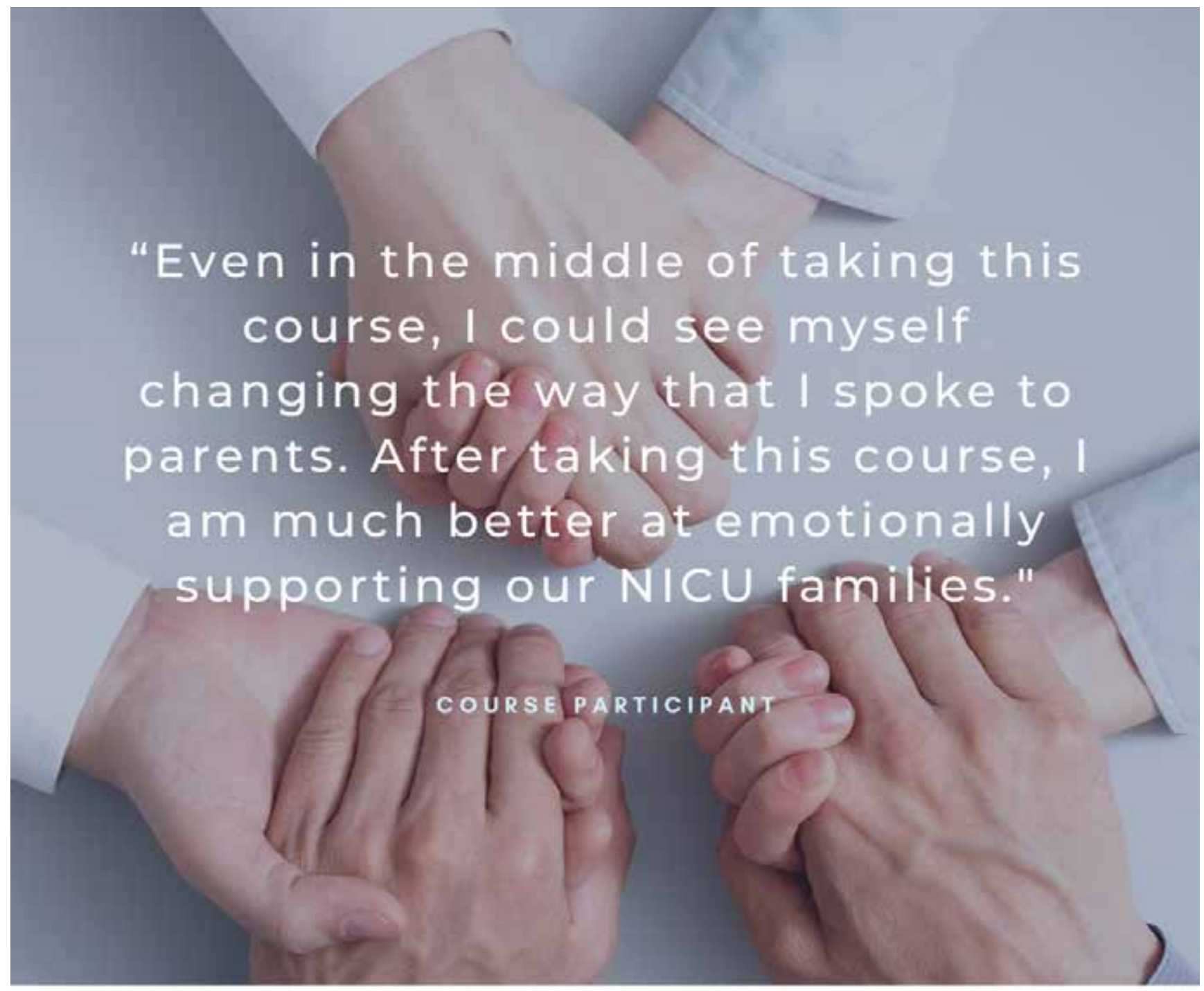

\section{Learn How Now}

validated online NICU staff education

WWW.MYNICUNETWORK.ORG
My NICU Network My Perinatal Network

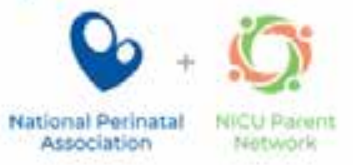

